\title{
Real Number Labelings for Paths and Cycles
}

\author{
Jerrold R. Griggs and Xiaohua Teresa jin
}

Abstract. The problem of radio channel assignments with multiple levels of interference depending on distance can be modelled using graph theory. The authors previously introduced a model of labeling by real numbers. Given a graph $G$, possibly infinite, and real numbers $k_{1}, k_{2} \geq 0$, an $L\left(k_{1}, k_{2}\right)$-labeling of $G$ assigns real numbers $f(x) \geq 0$ to the vertices $x$, such that the labels of vertices $u$ and $v$ differ by at least $k_{i}$ if $u$ and $v$ are at distance $i$ apart. We denote by $\lambda\left(G ; k_{1}, k_{2}\right)$ the infimum span over such labelings $f$. It is enough to determine $\lambda(G ; k, 1)$ for reals $k \geq 0$, which will be a continuous nondecreasing piecewise linear function. Here we present these functions for paths, cycles, and wheels.

\section{Introduction}

Hale (1980) used graph theory to model the efficient assignment of numerical channels to a network of transmitters such that interference between nearby transmitters is avoided [Hale 80]. Transmitters can be represented by vertices, with vertices for nearby transmitters joined by an edge. The goal is to minimize the span of the assignment between the longest and shortest channels. The resulting graph theory problems concern "generalized colorings," in which the colors are integers. Here the difference between integer labels is a concern, whereas in traditional coloring we only care whether vertices receive the same or different colors.

In the late 1980s Lanfear described to Roberts [Roberts 03] a channel assignment problem of this kind in which there are two levels of interference, depend-

(C) A K Peters, Ltd.

I542-795I/07 \$0.50 per page 
ing on the distance between the transmitters. In the basic problem, channels for close locations must be distinct (i.e., must differ by at least 1), while those for very close locations must differ by at least 2 (due to spectral spreading). Griggs introduced the analogous "lambda-labeling" problem for graphs and made the initial investigation of this graph theory problem with Yeh [Griggs and Yeh 92]. The generalization of the problem in which integer separations are specified at distances $1,2, \ldots, p$ was also introduced, and a sizable literature considering these labelings of graphs (see [Griggs 00, Calamoneri 06, Jin 05, Griggs and Jin 05, Griggs and Král', to appear] for overviews) has since appeared.

Over the past several years such problems have generated considerable attention due to the growth of large networks for wireless and mobile communications. Often, the transmitter towers are laid out in regular arrays that can be modeled effectively by the graph interpretation above. Since there is no reason why the channels and separations have to be restricted to integers, Griggs proposed a more general real number model for labeling graphs with distance conditions, which he has been investigating jointly with Xiaohua Teresa Jin [Griggs and Jin 06]. The purpose of this article is to present the optimal spans of labelings with arbitrary conditions at distance 2 for two fundamental graph families (paths and cycles) and a closely related family (wheels). In the real-number model, scaling allows us to reduce these problems for each graph to one with only a single parameter. These formulas will be useful in the future development of the theory of labelings with distance conditions.

For the remainder of this section, we shall review the basic definitions of realnumber labelings, along with the tools from the theory that will be helpful. For this paper, we restrict our attention to conditions at distance $p=2$, though the theory has been developed for general distance $p$. Section 2 collects our results and compares them with what is already in the literature. The following three sections contain the proofs of the formulas for paths, cycles, and wheels, respectively. The proofs are complicated, as is to be expected when one examines the surprisingly elaborate functions for the optimal spans. For instance, the formula for the $n$-cycle depends on $n$ modulo 12 for $n \geq 6$.

Let us fix a graph $G$, which we assume to be finite in this paper, although the theory considers infinite graphs as well. Let us also specify the separations, which are real numbers $k_{1}, k_{2} \geq 0$. We say an $L\left(k_{1}, k_{2}\right)$-labeling of $G$ is an assignment of real numbers $f(v)$ to the vertices $v$ of $G$, such that $|f(u)-f(v)| \geq k_{i}$ if $u$ and $v$ are at distance $i$ in $G$. We say that labeling $f$ belongs to the set $L\left(k_{1}, k_{2}\right)(G)$. We denote by $\lambda\left(G ; k_{1}, k_{2}\right)$ the infimum span over such $f$, where the span is the difference between the greatest and least labels $f(v)$.

Clearly, for finite $G$ such labelings exist for all $k_{1}, k_{2}$, and so the infimum, $\lambda\left(G ; k_{1}, k_{2}\right)$, exists as well. Griggs and Jin proved the existence of an optimal 
labeling of a nice form, in which all labels belong to the discrete set, denoted by $D\left(k_{1}, k_{2}\right)$, of linear combinations $\sum_{i} a_{i} k_{i}$, with nonnegative integer coefficients $a_{i}$ [Griggs and Jin 06].

Theorem I.I. (The $D$-Set Theorem (finite case, $p=2$ ).) [Griggs and Jin 06] Let $G=(V, E)$ be a finite graph. Let real numbers $k_{1}, k_{2} \geq 0$. There exists a finite optimal $L\left(k_{1}, k_{2}\right)$-labeling $f^{*}: V(G) \rightarrow[0, \infty)$ in which the smallest label is 0 , and all labels (and the span $\lambda\left(G ; k_{1}, k_{2}\right)$ ) belong to the set $D\left(k_{1}, k_{2}\right)$ such that the sum of coefficients $a_{1}+a_{2}<|V|$.

Due to the $D$-set Theorem, previous optimal integer labeling results are compatible with the theory of real-number labeling. An important property manifest in the setting of real-number labelings is scaling:

Proposition I.2. (Scaling Property.) For real numbers $d, k_{1}, k_{2} \geq 0$,

$$
\lambda\left(G ; d \cdot k_{1}, d \cdot k_{2}\right)=d \cdot \lambda\left(G ; k_{1}, k_{2}\right) .
$$

It is proven in [Griggs and Jin 06] that $\lambda\left(G ; k_{1}, k_{2}\right)$ is a continuous nondecreasing piecewise linear function of the real numbers $k_{i}$, where the pieces have nonnegative integer coefficients and where there are only finitely many pieces. The continuity means that it suffices to determine $\lambda\left(G ; k_{1}, k_{2}\right)$ for rational $k_{i}$ 's, and by scaling, it is enough to determine $\lambda\left(G ; k_{1}, k_{2}\right)$ for integer $k_{i}$ 's, which is the setting in which other researchers have worked. Indeed, our proofs here often reduce to the integer case. The analysis is more clear with the real-number model, however, and more results have emerged by considering real-number labelings. The theoretical results mentioned above give us additional tools.

Scaling implies, in particular, that for $k_{2}>0, \lambda\left(G, k_{1}, k_{2}\right)=k_{2} \lambda(G ; k, 1)$, where $k=k_{1} / k_{2}$. This reduces the two-parameter function to a one-parameter function, $\lambda(G ; k, 1), k \geq 0$. The results above ensure that this is a continuous nondecreasing piecewise linear function of $k$ with finitely many pieces. Further, each piece has the form $a k+b$ for some integers $a, b \geq 0$. It is this function that we will obtain for

1. paths $P_{n}$ on $n$ vertices;

2. cycles $C_{n}$ on $n \geq 3$ vertices; and

3. wheels $W_{n}, n \geq 3$, consisting of a cycle $C_{n}$ and a vertex adjacent to all of its vertices (see Figure 3 ). 
In their original paper on distance labeling, Griggs and Yeh [Griggs and Yeh 92] worked out the (2,1)-labeling number for paths and cycles, showing that $\lambda\left(C_{n} ; 2,1\right)=4$ for all $n$, which is the same value as $\lambda\left(P_{n} ; 2,1\right)$ for all $n \geq 5$.

While working out the basics of the general theory of real-number labeling with distance conditions, the authors determined $\lambda\left(P_{n} ; k, 1\right)$ and $\lambda\left(C_{n} ; k, 1\right)$ for arbitrary real $k \geq 0$. This was in 2003. But it took longer to work out the basics of the theory, which needed to be in the first paper of the series, see [Griggs and Jin 06]. In reviewing past work on integer labelings with distance conditions, the authors discovered that Georges and Mauro had already determined the values $\lambda\left(P_{n} ; k_{1}, k_{2}\right)$ and $\lambda\left(C_{n} ; k_{1}, k_{2}\right)$ for integers $k_{1} \geq k_{2}$ [Georges and Mauro 95]. Their formulas are given in terms of the ratio $k_{1} / k_{2}$, which makes sense in view of the reduction above to the one-parameter values $\lambda\left(P_{n} ; k, 1\right)$ and $\lambda\left(C_{n} ; k, 1\right)$ for $k \geq 1$.

Thus, our formulas for paths and cycles can be deduced from the existing theory for $k \geq 1$. We place them in the context of real-number labelings, where the formulas are more illuminating. Because our proofs use different methods, which will be useful for other graphs in the future, we present them in detail. Further, we offer new results, by expanding the formulas to allow $k_{1}<k_{2}$ (that is, $k \geq 0$ ). We also obtain new formulas, solving the wheels $W_{n}$.

For actual networks, the frequency channel separations $k_{i}$ for two transmitters are often inversely proportional to the distance $i$ between them [Bertossi et al. 03] Most articles assume that the separations are nonincreasing, $k_{1} \geq k_{2}$. But this is not required in our theory, and there are different settings for which these labelings are a good model, but without the added assumption on the separations $k_{i}$ (see [Griggs and Jin 08]).

For additional motivation for studying paths and cycles, we mention these network models:

- A typical $n$-cell linear highway cellular system [Anand et al. 03] along a highway (with the base-stations/transmitters in the center of each cell) can be modeled by a path $P_{n}$.

- A loop cellular system around a big city [Anand et al. 03, Bertossi et al. 03], due to high buildings, can be modeled by a cycle $C_{n}$.

Also, paths and cycles are induced subgraphs of many graphs, such as those that occur in typical cellular systems. 


\section{Results on Paths, Cycles, and Wheels.}

Here are our results:

Theorem 2.I. (Path Theorem.) For real $k \geq 0$, we have

$$
\begin{aligned}
& \lambda\left(P_{2} ; k, 1\right)=k, \\
& \lambda\left(P_{3} ; k, 1\right)= \begin{cases}1 & \text { if } 0 \leq k \leq \frac{1}{2} \\
2 k & \text { if } \frac{1}{2} \leq k \leq 1 \\
k+1 & \text { if } k \geq 1,\end{cases} \\
& \lambda\left(P_{4} ; k, 1\right)=k+1, \\
& \lambda\left(P_{5} ; k, 1\right)=\lambda\left(P_{6} ; k, 1\right)=\left\{\begin{array}{lll}
k+1 & \text { if } 0 \leq k \leq 1 \\
2 k & \text { if } 1 \leq k \leq 2 \\
k+2 & \text { if } k \geq 2 .
\end{array}\right.
\end{aligned}
$$

For $n \geq 7$, we have

$$
\lambda\left(P_{n} ; k, 1\right)=\left\{\begin{array}{lll}
k+1 & \text { if } \quad 0 \leq k \leq \frac{1}{2} \\
3 k & \text { if } \frac{1}{2} \leq k \leq \frac{2}{3} \\
2 & \text { if } \quad \frac{2}{3} \leq k \leq 1 \\
2 k & \text { if } 1 \leq k \leq 2 \\
k+2 & \text { if } k \geq 2 .
\end{array}\right.
$$

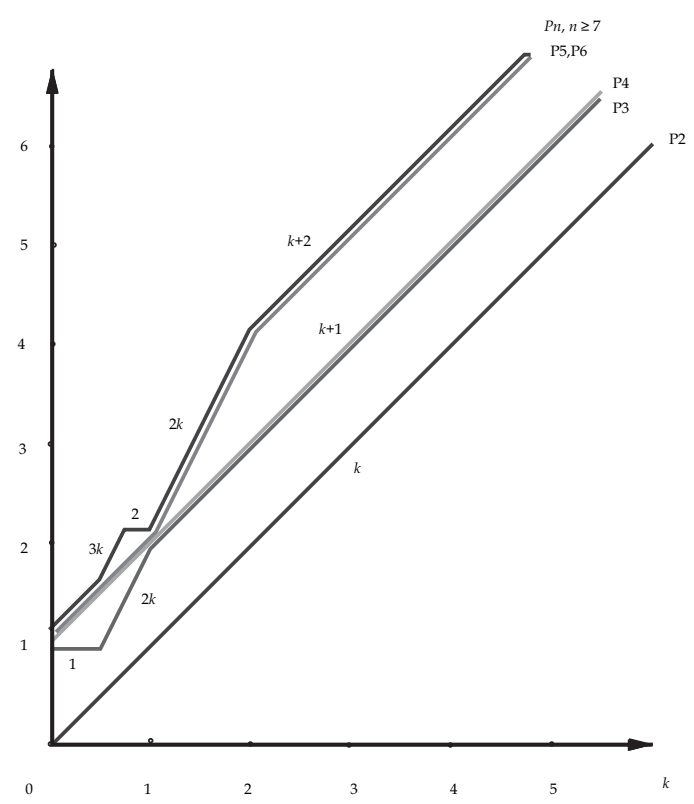

Figure I. The minimum $\operatorname{span} \lambda\left(P_{n} ; k, 1\right)$. 
Theorem 2.2. (Cycle Theorem.) For real $k \geq 0$, we have

$$
\begin{aligned}
& \lambda\left(C_{3} ; k, 1\right)=2 k, \\
& \lambda\left(C_{4} ; k, 1\right)= \begin{cases}k+1 & \text { if } 0 \leq k \leq \frac{1}{2} \\
3 k & \text { if } \frac{1}{2} \leq k \leq 1 \\
k+2 & \text { if } k \geq 1,\end{cases} \\
& \lambda\left(C_{5} ; k, 1\right)=\left\{\begin{array}{lll}
2 & \text { if } \quad 0 \leq k \leq \frac{1}{2} \\
4 k & \text { if } \frac{1}{2} \leq k \leq 1 \\
4 & \text { if } 1 \leq k \leq 2 \\
2 k & \text { if } \quad k \geq 2 .
\end{array}\right.
\end{aligned}
$$

For $n \geq 6$, the values of $\lambda\left(C_{n}, k, 1\right)$ are indicated in the following tables:

\begin{tabular}{|l|r|r|}
\hline$\lambda\left(C_{n} ; k, 1\right)$ & $n \equiv 0(\bmod 4)$ & $n \neq \equiv 0(\bmod 4)$ \\
\hline if $0 \leq k \leq \frac{1}{2}$ & $k+1$ & 2 \\
\hline if $\frac{1}{2} \leq k \leq \frac{2}{3}$ & $3 k$ & 2 \\
\hline
\end{tabular}

\begin{tabular}{|l|r|r|}
\hline$\lambda\left(C_{n} ; k, 1\right)$ & $n \equiv 0(\bmod 3)$ & $n \not \equiv 0(\bmod 3)$ \\
\hline if $\frac{2}{3} \leq k \leq 1$ & 2 & $3 k$ \\
\hline if $1 \leq k \leq 2$ & $2 k$ & $k+2$ \\
\hline
\end{tabular}

\begin{tabular}{|l|r|r|r|}
\hline$\lambda\left(C_{n} ; k, 1\right)$ & $n \equiv 0(\bmod 4)$ & $n \equiv 2(\bmod 4)$ & $n \equiv 1$ or $3(\bmod 4)$ \\
\hline if $2 \leq k \leq 3$ & $k+2$ & $2 k$ & $2 k$ \\
\hline if $k \geq 3$ & $k+2$ & $k+3$ & $2 k$ \\
\hline
\end{tabular}
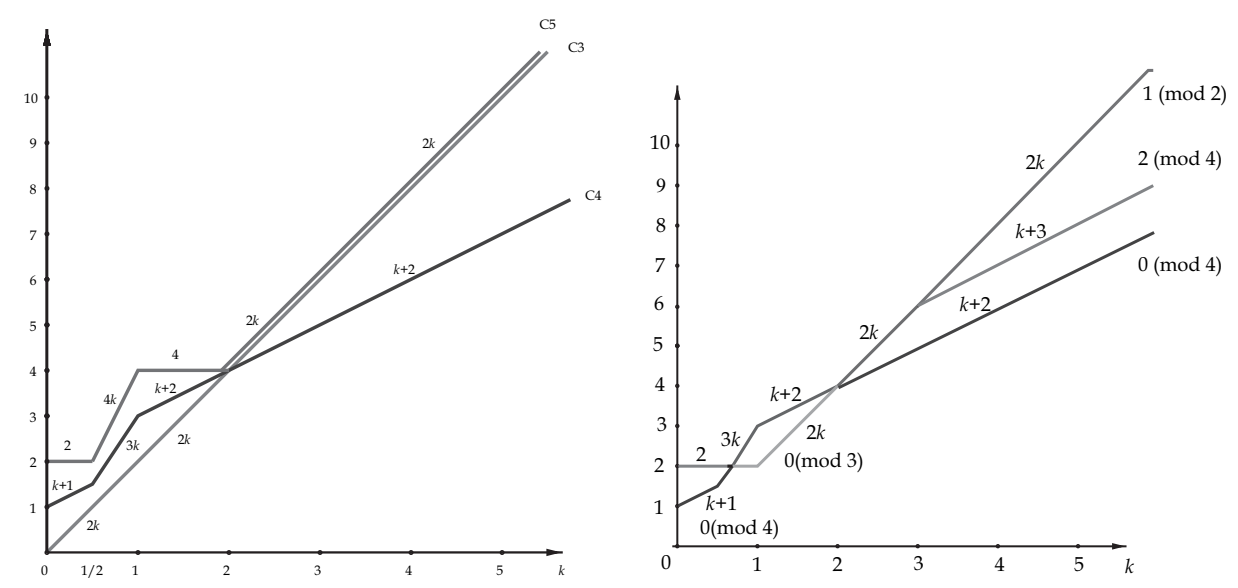

Figure 2. The minimum $\operatorname{span} \lambda\left(C_{n} ; k, 1\right)$ for $n=3,4,5$ and $n \geq 6$. 
Theorem 2.3. (Wheel Theorem.) For real $k \geq 0$, we have

$$
\begin{aligned}
& \lambda\left(W_{3} ; k, 1\right)=3 k, \\
& \lambda\left(W_{4} ; k, 1\right)= \begin{cases}k+1 & \text { if } \quad 0 \leq k \leq \frac{1}{3} \\
4 k & \text { if } \quad \frac{1}{3} \leq k \leq 1 \\
2 k+2 & \text { if } \quad k \geq 1 .\end{cases}
\end{aligned}
$$

For odd $n \geq 5$, we have

$$
\lambda\left(W_{n} ; k, 1\right)= \begin{cases}\frac{n-1}{2} & \text { if } 0 \leq k \leq \frac{1}{3} \\ 3 k+\frac{n-3}{2} & \text { if } \frac{1}{3} \leq k \leq \frac{1}{2} \\ n k & \text { if } \frac{1}{2} \leq k \leq 1 \\ k+n-1 & \text { if } 1 \leq k \leq \frac{n-1}{2} \\ 3 k & \text { if } k \geq \frac{n-1}{2} .\end{cases}
$$

For even $n \geq 5$, we have

$$
\lambda\left(W_{n} ; k, 1\right)= \begin{cases}k+\frac{n}{2}-1 & \text { if } 0 \leq k \leq \frac{1}{3} \\ 4 k+\frac{n}{2}-2 & \text { if } \frac{1}{3} \leq k \leq \frac{1}{2} \\ n k & \text { if } \frac{1}{2} \leq k \leq 1 \\ k+n-1 & \text { if } 1 \leq k \leq \frac{n}{2}-1 \\ 2 k+\frac{n}{2} & \text { if } k \geq \frac{n}{2}-1 .\end{cases}
$$
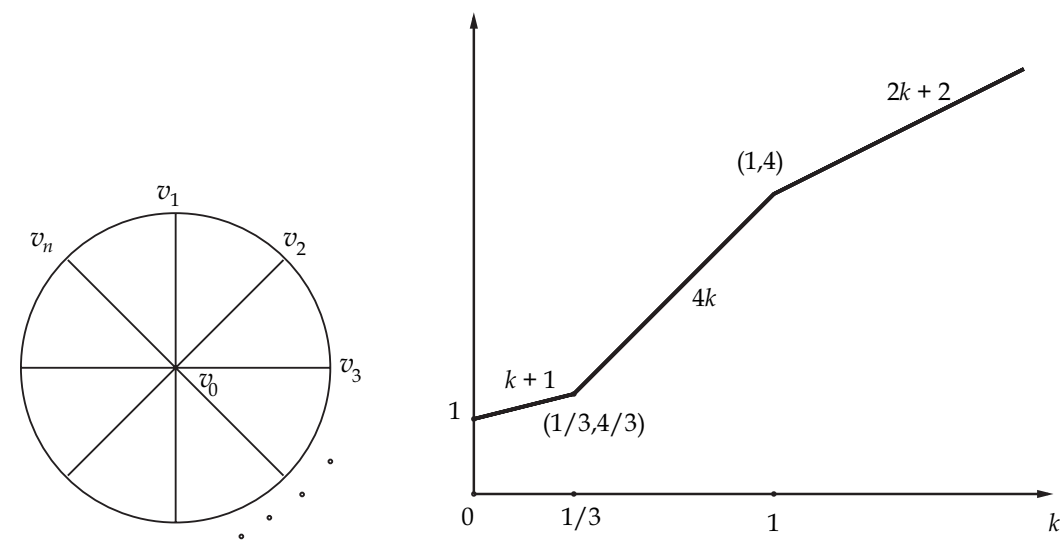

Figure 3. Wheel $W_{n}, n \geq 3$ (left), and $\lambda\left(W_{4} ; k, 1\right)$ (right). 

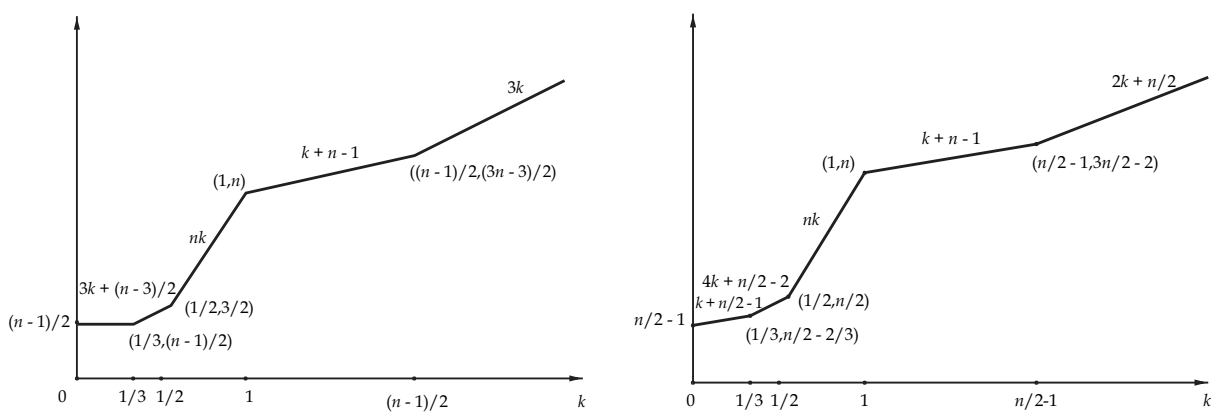

Figure 4. $\lambda\left(W_{n} ; k, 1\right)$ for odd $n \geq 5$ (left), and for even $n \geq 6$ (right).

\section{The Proof for Paths}

Let the vertices of path $P_{n}$ be called, in order starting from one end, $v_{1}, \ldots, v_{n}$. The result is immediate for $P_{2}$, which is a single edge. Next, consider $P_{3}$.

Proposition 3.I. For real $k \geq 0$, we have

$$
\lambda\left(P_{3} ; k, 1\right)= \begin{cases}1 & \text { if } \quad 0 \leq k \leq \frac{1}{2} \\ 2 k & \text { if } \frac{1}{2} \leq k \leq 1 \\ k+1 & \text { if } k \geq 1\end{cases}
$$

Proof. The upper bound is attained by labeling $f$ with

$$
\left(f\left(v_{1}\right), f\left(v_{2}\right), f\left(v_{3}\right)\right)= \begin{cases}(0, k, 1) & \text { if } \quad 0 \leq k \leq \frac{1}{2} \\ (0, k, 2 k) & \text { if } \frac{1}{2} \leq k \leq 1 \\ (0, k+1,1) & \text { if } k \geq 1\end{cases}
$$

We will show that is the lower bound, too. Assume $f$ is an optimal labeling with least label 0 , as in the $D$-Set Theorem. By the condition at distance 2, $f\left(v_{1}\right)$ or $f\left(v_{3}\right) \geq 1$. Hence, $\lambda\left(P_{3} ; k, 1\right) \geq 1$, which gives the desired bound for $0 \leq k \leq \frac{1}{2}$.

Claim 3.2. For $\frac{1}{2} \leq k \leq 1, \lambda\left(P_{3} ; k, 1\right) \geq 2 k$.

Proof. The labels for any two of the three vertices of $P_{3}$ must differ by at least $k$ in this range, and so the span of any labeling must be at least $2 k$, proving the claim. 
Claim 3.3. For $k \geq 1, \lambda\left(P_{3} ; k, 1\right) \geq k+1$.

Proof. Assume $\lambda\left(P_{3} ; k, 1\right)=l<k+1$. By the $D$-Set Theorem, we may assume that at least two of the three labels are less than $k$ (otherwise, we may replace $f(v)$ by $l-f(v)$ for all vertices $v)$. By the distance conditions, labels less than $k$ cannot be adjacent, so $f\left(v_{1}\right), f\left(v_{3}\right)<k$. By the condition at distance $2, f\left(v_{1}\right)$ or $f\left(v_{3}\right) \geq 1$. Hence, $f\left(v_{2}\right) \geq k+1$, which contradicts the assumption, proving the claim.

Proposition 3.4. For real $k \geq 0$, we have $\lambda\left(P_{4} ; k, 1\right)=k+1$.

Proof. The upper bound is attained by labeling $\left(f\left(v_{1}\right), f\left(v_{2}\right), f\left(v_{3}\right), f\left(v_{4}\right)\right)=$ $(k, 0, k+1,1)$. We will show that is also the lower bound.

For $k \geq 1$, we have $\lambda\left(P_{4} ; k, 1\right) \geq \lambda\left(P_{3} ; k, 1\right)=k+1$, as desired. It remains to treat small $k$. It suffices to prove the lower bound of $k+1$ for $0<k<1$, since it follows at $k=0$ by continuity of $\lambda$.

Claim 3.5. For $0<k<1$, we have $\lambda\left(P_{4} ; k, 1\right) \geq k+1$.

Proof. Assume to the contrary that, for some such $k, l=\lambda\left(P_{4} ; k, 1\right)<k+1$, and let $f$ be an optimal labeling as in the $D$-Set Theorem.

Suppose $f\left(v_{2}\right)<1$. If $f\left(v_{2}\right)=0$, then by the distance conditions, both $f\left(v_{1}\right), f\left(v_{3}\right) \geq k$, and the greater of the two must then be at least $k+1$, contradicting the assumption on $l$. So $f\left(v_{2}\right)$ must of the form $i k$ for some integer $i>0$ (since it is in $D(k, 1)$ and is less than 1 ). Then $f\left(v_{4}\right)$ must be at least $i k+1 \geq k+1$, again contradicting the assumption.

Hence, $f\left(v_{2}\right) \geq 1$. Now we define a complementary labeling $f^{\prime}$ by $f^{\prime}(v)=$ $l-f(v)$. While $f^{\prime}$ is also an optimal $L(k, 1)$-labeling, it may not be one as in the $D$-Set Theorem (with all labels in $D(k, 1)$ ). But we can obtain such a labeling, call it $f^{\prime \prime}$, as in the proof of the $D$-Set Theorem [Griggs and Jin 06]: For each $v$, let $f^{\prime \prime}(v)$ be the largest element of $D(k, 1)$ that is at most $f^{\prime}(v)$. Now we have that $f^{\prime \prime}(v) \leq f^{\prime}(v)=l-f(v) \leq l-1<k<1$, and we get a contradiction the same way as before, proving the claim and the proposition.

Proposition 3.6. For real $k \geq 0$, we have

$$
\lambda\left(P_{5} ; k, 1\right)=\lambda\left(P_{6} ; k, 1\right)=\left\{\begin{array}{lll}
k+1 & \text { if } 0 \leq k \leq 1 \\
2 k & \text { if } 1 \leq k \leq 2 \\
k+2 & \text { if } k \geq 2
\end{array}\right.
$$


Proof. Both graphs have the same spans. For the upper bound, it is enough to show how to label $P_{6}$ :

$$
\begin{cases}(k+1, k, 0, k+1,1,0) & \text { if } \quad 0 \leq k \leq 1 \\ (0, k, 2 k, 0, k, 2 k) & \text { if } \quad 1 \leq k \leq 2 \\ (0, k+1,1, k+2,0, k+1) & \text { if } \quad k \geq 2\end{cases}
$$

It remains to prove the lower bound on $P_{5}$. For $0 \leq k \leq 1$ we use $\lambda\left(P_{5} ; k, 1\right) \geq$ $\lambda\left(P_{4} ; k, 1\right)=k+1$. Next consider $k$ between 1 and 2 .

Claim 3.7. For $1 \leq k \leq 2, \lambda\left(P_{5} ; k, 1\right) \geq 2 k$.

Proof. Assume $l=\lambda\left(P_{5} ; k, 1\right)<2 k$, and let $f$ be an optimal labeling as in the $D$-Set Theorem. We may assume that at least two of the three labels $f\left(v_{2}\right), f\left(v_{3}\right), f\left(v_{4}\right)$ are less than $k$ (or else take a complementary labeling $f^{\prime \prime}$ as in the proof of Claim 3). By the distance conditions, these two labels cannot be adjacent, so $f\left(v_{2}\right)$ and $f\left(v_{4}\right)$ are both less than $k$ and at least 1 apart. The greater of the two labels, say $f\left(v_{2}\right)$, then satisfies $1 \leq f\left(v_{2}\right)<k$. So $f\left(v_{1}\right), f\left(v_{3}\right) \geq k+1$. By the condition at distance $2, f\left(v_{1}\right)$ or $f\left(v_{3}\right) \geq k+2 \geq 2 k$, contradicting $l<2 k$, and the claim follows.

Claim 3.8. For $k \geq 2, \lambda\left(P_{5} ; k, 1\right) \geq k+2$.

Proof. Assume to the contrary that $l=\lambda\left(P_{5} ; k, 1\right)<k+2$, and let $f$ be an optimal labeling as in the $D$-Set Theorem. Following the proof of Claim 4 again leads to $f\left(v_{1}\right)$ or $f\left(v_{3}\right) \geq k+2$, a contradiction, proving the claim and proposition.

Proposition 3.9. Let $n \geq 7$. For real $k \geq 0$, we have

$$
\lambda\left(P_{n} ; k, 1\right)= \begin{cases}k+1 & \text { if } 0 \leq k \leq \frac{1}{2} \\ 3 k & \text { if } \frac{1}{2} \leq k \leq \frac{2}{3} \\ 2 & \text { if } \frac{2}{3} \leq k \leq 1 \\ 2 k & \text { if } 1 \leq k \leq 2 \\ k+2 & \text { if } k \geq 2 .\end{cases}
$$


Proof. According to the value of $k$, we repeat an underlined pattern until all of $P_{n}$ is labeled to achieve the stated optimal spans:

$$
\left\{\begin{array}{lll}
(\underline{0, k+1,1, k}, \ldots) & \text { if } \quad 0 \leq k \leq \frac{1}{2} \\
(\underline{0, k, 2 k, 3 k}, \ldots) & \text { if } \frac{1}{2} \leq k \leq \frac{2}{3} \\
(\overline{0,1,2, \ldots)} & \text { if } \frac{2}{3} \leq k \leq 1 \\
(\underline{0, k, 2 k}, \ldots) & \text { if } 1 \leq k \leq 2 \\
(\underline{0, k+1,1, k+2}, \ldots) & \text { if } \quad k \geq 2 .
\end{array}\right.
$$

The lower bounds follow from those for $P_{5}$, except in the range $\frac{1}{2}<k<1$. Next, one can easily check that for $\frac{1}{2}<k \leq \frac{2}{3},(k+1,3 k) \cap D(k, 1)=\emptyset$, and for $\frac{2}{3}<k<1,(k+1,2) \cap D(k, 1)=\emptyset$. By the $D$-Set Theorem, it then suffices to prove the next claim.

Claim 3.10. For $\frac{1}{2}<k<1, \lambda\left(P_{7} ; k, 1\right)>k+1$.

Proof. Assume for some such $k$ that $l=\lambda\left(P_{7} ; k, 1\right) \leq k+1$, and let $f$ be an optimal labeling as in the $D$-Set Theorem. We may assume that at least two of the three labels $f\left(v_{3}\right), f\left(v_{4}\right), f\left(v_{5}\right)$ are less than 1 (or else take a complementary labeling $f^{\prime \prime}$ as in the proof of Claim 3.5). These two labels cannot be at distance 2, so we may assume they are at $v_{3}$ and $v_{4}$ (or else reverse the order of the vertices on $\left.P_{7}\right)$. We only need to work now on $v_{1}$ through $v_{6}$. By symmetry, we may assume $f\left(v_{3}\right)>f\left(v_{4}\right)$, so that $f\left(v_{3}\right) \geq k$.

Since $f\left(v_{3}\right)<1$, the condition at distance 2 forces $f\left(v_{1}\right) \geq f\left(v_{3}\right)+1 \geq k+1$. Due to the span of $f$, it must be that $f\left(v_{1}\right)=k+1$, which forces $f\left(v_{3}\right)=k$ and $f\left(v_{4}\right)=0$. Then $v_{1}$ forces $f\left(v_{2}\right) \leq 1$, while $v_{3}$ and $v_{4}$ force $f\left(v_{2}\right)>1$, a contradiction, proving the claim and proposition.

This completes the proof of Theorem 2.1.

\section{The Proof for Cycles}

Let the vertices of cycle $C_{n}$ be called, in order going around, $v_{1}, \ldots, v_{n}$. The result is almost immediate for $C_{3}$, for which the optimal labeling is $(0, k, 2 k)$. We take care of $C_{4}$ and $C_{5}$ in the next two propositions.

Proposition 4.I. For real $k \geq 0$, we have

$$
\lambda\left(C_{4} ; k, 1\right)= \begin{cases}k+1 & \text { if } \quad 0 \leq k \leq \frac{1}{2} \\ 3 k & \text { if } \frac{1}{2} \leq k \leq 1 \\ k+2 & \text { if } \quad k \geq 1\end{cases}
$$


Proof. The upper bound is attained by the labeling

$$
\left(f\left(v_{1}\right), f\left(v_{2}\right), f\left(v_{3}\right), f\left(v_{4}\right)\right)= \begin{cases}(0, k, 1, k+1) & \text { if } 0 \leq k \leq \frac{1}{2} \\ (0, k, 2 k, 3 k) & \text { if } \quad \frac{1}{2} \leq k \leq 1 \\ (0, k+1,1, k+2) & \text { if } \quad k \geq 1\end{cases}
$$

For the lower bound, consider an optimal labeling $f$ as described by the $D$-Set Theorem. Suppose $f\left(v_{1}\right)=0$. The distance conditions force $f\left(v_{2}\right), f\left(v_{3}\right), f\left(v_{4}\right) \geq$ $\min \{k, 1\}$. The span among $\left\{f\left(v_{2}\right), f\left(v_{3}\right), f\left(v_{4}\right)\right\}$ is at least $\lambda\left(P_{3} ; k, 1\right)$, so $\lambda\left(C_{4} ; k, 1\right) \geq \lambda\left(P_{3} ; k, 1\right)+\min \{k, 1\}$. Applying Theorem 2.1 to evaluate this, we obtain the stated bound.

Proposition 4.2. For real $k \geq 0$, we have

$$
\lambda\left(C_{5} ; k, 1\right)= \begin{cases}2 & \text { if } 0 \leq k \leq \frac{1}{2} \\ 4 k & \text { if } \frac{1}{2} \leq k \leq 1 \\ 4 & \text { if } 1 \leq k \leq 2 \\ 2 k & \text { if } k \geq 2\end{cases}
$$

Proof. The upper bound is attained by labeling

$$
\left\{\begin{array}{lll}
(0, k, 1, k+1,2) & \text { if } \quad 0 \leq k \leq \frac{1}{2} \\
(0, k, 2 k, 3 k, 4 k) & \text { if } \quad \frac{1}{2} \leq k \leq 1 \\
(0,2,4,1,3) & \text { if } \quad 1 \leq k \leq 2 \\
(0, k, 2 k, 1, k+1) & \text { if } \quad k \geq 2
\end{array}\right.
$$

We will show that is the lower bound, too. Assume $f$ is an optimal labeling as in the $D$-Set Theorem.

Claim 4.3. For $0 \leq k \leq \frac{1}{2}$, we have $\lambda\left(C_{5} ; k, 1\right) \geq 2$.

Proof. For any three vertices of $C_{5}$, some two are at distance 2, so at most two vertices have labels $f(v)$ in $[0,1)$. Similarly, at most two vertices have labels in $[1,2)$. So some vertex must have label at least 2 , and the span of $f$ is at least 2, proving the claim.

Claim 4.4. For $\frac{1}{2} \leq k \leq 1$, we have $\lambda\left(C_{5} ; k, 1\right) \geq 4 k$, and for $1 \leq k \leq 2$, we have $\lambda\left(C_{5} ; k, 1\right) \geq 4$. 
Proof. Suppose $\frac{1}{2} \leq k \leq 1$. The distance conditions imply that any two labels differ by at least $k$, and so the span of $f$ is at least $4 k$, as claimed. For $k \geq 1$, we then have $\lambda\left(C_{5} ; k, 1\right) \geq \lambda\left(C_{5} ; 1,1\right)=4$, proving the claim.

Claim 4.5. For $k \geq 2$, we have $\lambda\left(C_{5} ; k, 1\right) \geq 2 k$.

Proof. Of any three vertices, some two are adjacent. Thus, the conditions at distance 1 imply that at most two vertices have labels $f(v)$ in $[0, k)$. Similarly, at most two vertices have labels in $[k, 2 k)$. So some vertex must have label at least $2 k$, and the span of $f$ is at least $2 k$. This proves the claim and proposition.

It remains to treat $C_{n}$ for all $n \geq 6$. The first proposition gives labelings that achieve the stated bounds.

Proposition 4.6. Let $n \geq 6$. For real $k \geq 0$, there are labelings that achieve the bounds in the Cycle Theorem.

Proof. Here are the labelings, depending on the value of $k$ and on $n \bmod 3$ and mod 4. The underlined sections are repeated as many times as needed.

For $0 \leq k \leq \frac{1}{2}$,

$$
\begin{cases}(0, k, 1, k+1) & \text { if } n \equiv 0(\bmod 4) \\ (0,1,2,0,1,2,0,1,2, \underline{0, k, 1,2)}) & \text { if } n \equiv 1(\bmod 4) \\ (0,1,2,0,1,2, \underline{0, k, 1,2)} & \text { if } n \equiv 2(\bmod 4) \\ (0,1,2, \underline{0, k, 1,2)} & \text { if } n \equiv 3(\bmod 4) .\end{cases}
$$

For $\frac{1}{2} \leq k \leq \frac{2}{3}$,

$$
\left\{\begin{array}{lll}
(0, k, 2 k, 3 k) & \text { if } & n \equiv 0(\bmod 4) \\
(0,1,2,0,1,2,0,1,2, \underline{0, k, 2 k, 2)}) & \text { if } & n \equiv 1(\bmod 4) \\
(0,1,2,0,1,2, \underline{0, k, 2 k, 2)} & \text { if } & n \equiv 2(\bmod 4) \\
(0,1,2, \underline{0, k, 2 k, 2)}) & \text { if } & n \equiv 3(\bmod 4) .
\end{array}\right.
$$

For $\frac{2}{3} \leq k \leq 1$,

$$
\begin{cases}(\underline{0,1,2}) & \text { if } n \equiv 0(\bmod 3) \\ (0, k, 2 k, 3 k, 0,1,2) & \text { if } n \equiv 1(\bmod 3) \\ (0, k, 2 k, 3 k, 0, k, 2 k, 3 k, \underline{0,1,2)}) & \text { if } n \equiv 2(\bmod 3) .\end{cases}
$$


For $1 \leq k \leq 2$,

$$
\begin{cases}(\underline{0, k, 2 k}) & \text { if } n \equiv 0(\bmod 3) \\ (0, k+1,1, k+2, \underline{0, k, k+2}) & \text { if } n \equiv 1(\bmod 3) \\ (0, k+1,1, k+2,0, k+1,1, k+2, \underline{0, k, k+2)} & \text { if } n \equiv 2(\bmod 3) .\end{cases}
$$

For $2 \leq k \leq 3$,

$$
\begin{cases}(0, k+1,1, k+2) & \text { if } n \equiv 0(\bmod 4) \\ (\overline{0, k, 2 k, 0, k, 2 k, 0, k, 2 k, 0, k+1,1, k+2)}) & \text { if } n \equiv 1(\bmod 4) \\ (0, k, 2 k, 0, k, 2 k, \underline{0, k+1,1, k+2)} & \text { if } n \equiv 2(\bmod 4) \\ (0, k, 2 k, 0, k+1,1, k+2) & \text { if } n \equiv 3(\bmod 4) .\end{cases}
$$

For $k \geq 3$,

$$
\begin{cases}(\underline{0, k+1,1, k+2)}) & \text { if } n \equiv 0(\bmod 4) \\ (0, k, 2 k, 0, k, 2 k, 0, k, 2 k, 0, k+1,1, k+2) & \text { if } n \equiv 1(\bmod 4) \\ (0, k+1,1, k+2,2, k+3, \underline{0, k+1,1, k+2)} & \text { if } n \equiv 2(\bmod 4) \\ (0, k, 2 k, 0, k+1,1, k+2) & \text { if } n \equiv 3(\bmod 4) .\end{cases}
$$

It remains to prove the lower bounds for $n \geq 6$.

Let $f$ be an optimal labeling as in the $D$-Set Theorem. We begin with $k \leq \frac{2}{3}$, which splits into cases according to $n(\bmod 4)$. If $n \equiv 0(\bmod 4)$, then $n \geq 8$, and $C_{n}$ contains an induced $P_{7}$. Thus, $\lambda\left(C_{n} ; k, 1\right) \geq \lambda\left(P_{7} ; k, 1\right)$, which is the desired formula, $k+1$ for $0 \leq k \leq \frac{1}{2}$ and $3 k$ for $\frac{1}{2} \leq k \leq \frac{2}{3}$. We now treat the other values of $n$.

Proposition 4.7. Let $n \geq 6$ with $n \not \equiv 0(\bmod 4)$. For $0 \leq k \leq \frac{2}{3}$, the spans stated in the Cycle Theorem cannot be improved.

Proof. It suffices to prove the lower bound, 2, at $k=0$. Assume to the contrary that for some such $n, l=\lambda\left(C_{n} ; 0,1\right)<2$. Then each vertex $v_{i}$ has its label $f\left(v_{i}\right)$ either in the interval $[0,1)$ or the interval $[1,2)$. Since no two vertices at distance 2 can have labels in the same unit interval, it must be that the labels going around $C_{n}$ have two in $[0,1)$ followed by two in $[1,2)$ followed by two in $[0,1)$ again, and so on. But this is possible only if 4 divides $n$, a contradiction.

We next treat $\frac{2}{3} \leq k \leq 1$. If $n \geq 8$, then $C_{n}$ contains an induced $P_{7}$ as above, and the value of $\lambda\left(P_{7} ; k, 1\right)=2$ is a lower bound. For $n \equiv 0(\bmod 3)$, this is the desired bound, though we must still prove it for $C_{6}$ : The vertices $v_{1}, v_{3}, v_{5}$ in $C_{6}$ are pairwise at distance two, so require span at least 2 . This still leaves $n \not \equiv 0(\bmod 3)$ for this range in $k$. 
Proposition 4.8. Let $n \geq 7$ with $n \not \equiv 0(\bmod 3)$. For $\frac{2}{3} \leq k \leq 1$, we have that $\lambda\left(C_{n} ; k, 1\right) \geq 3 k$.

Proof. Assume to the contrary that for some such $n$ and $k$ we have $\lambda\left(C_{n} ; k, 1\right)<3 k$. Every label $f\left(v_{i}\right)$ is in one of the intervals $[0, k),[k, 2 k),[2 k, 3 k)$, and no two labels within distance 2 are in the same interval. Hence, each of these intervals contains labels for at most $\lfloor n / 3\rfloor$ of the vertices. But this is less than $n / 3$, since $n \not \equiv 0(\bmod 3)$, so we have not accounted for all $n$ vertices, a contradiction.

The next range up is $1 \leq k \leq 2$. The lower bound here for cycles $C_{n}, n \geq 6$ with $n \equiv 0(\bmod 3)$, follows immediately from the span of $P_{5}$, which is $2 k$ in this range. We then need to treat the remaining $n$.

Proposition 4.9. Let $n \geq 6$ with $n \not \equiv 0(\bmod 3)$. For $1 \leq k \leq 2$, we have $\lambda\left(C_{n} ; k, 1\right) \geq$ $k+2$.

Proof. No two labels less than 1 can be within distance 2 of each other, so there are at most $\lfloor n / 3\rfloor<n / 3$ such labels. Thus, there exist some three consecutive vertices with labels at least 1 . Using the fact that $\lambda\left(P_{3} ; k, 1\right)=k+1$, we get that the span of $f$ is at least $k+2$.

We now treat the large values, $k \geq 2$. Again, we get a lower bound from the span of $P_{5}$, which is $k+2$ in this range. It is the bound we want for $n \equiv 0(\bmod 4)$. For other $n \geq 6$ we must do better.

Proposition 4.10. Let $n \geq 6$ with $n \not \equiv 0(\bmod 4)$. If $n$ is odd, then we have $\lambda\left(C_{n} ; k, 1\right) \geq 2 k$ for $k \geq 2$. If $n$ is even, then we have $\lambda\left(C_{n} ; k, 1\right) \geq 2 k$ for $2 \leq k \leq 3$ and $\lambda\left(C_{n} ; k, 1\right) \geq k+3$ for $k \geq 3$.

Proof. First suppose $n \geq 6$ is odd and $k \geq 2$. No two adjacent vertices have labels less than $k$ apart, so the number of vertices with labels in $[0, k)$ is at most $\lfloor n / 2\rfloor<n / 2$, and the same is true for labels in $[k, 2 k)$. Hence, some vertex has label at least $2 k$, so the span of $f$ is at least $2 k$.

Then suppose $n \geq 6$ is even, but $\not \equiv 0(\bmod 4)$, say $n=2 r$ where $r$ is odd. We must show that $\lambda\left(C_{n} ; k, 1\right) \geq \min \{2 k, k+3\}$ for $k \geq 2$. Suppose not, say $l=\lambda\left(C_{n} ; k, 1\right)<\min \{2 k, k+3\}$. Arguing as for odd $n$, we find that there are $n / 2$ labels each in the intervals $[0, k)$ and $[k, l]$ (as $l<2 k$ ), and they alternate between the two intervals. Looking at the $r$ labels in $[0, k)$ in order going around, we find that consecutive ones, which are at distance 2 in $C_{n}$, differ by at least 1. Since $r$ is odd, some label in $[0, k)$ is at least 2 . Its two neighbors on $C_{n}$ have "large labels" (at least $k$ ). The distance conditions mean that each neighbor has 
label at least $k+2$, and the greater of the two must then be at least $k+3$. So the span of $f$ is at least $k+3$, which contradicts the assumption on $l$.

This completes the proof of Theorem 2.2.

\section{The Proof for Wheels}

While the wheel $W_{n}$ is closely related to the cycle $C_{n}$, note that the extra vertex brings the diameter down to just 2 , which clearly affects distance labelings. In fact, for an $L(k, 1)$-labeling, any two of the $n+1$ labels must differ by at least $\min \{k, 1\}$.

As before, we denote the vertices going around the $n$-cycle in $C_{n}$ by $v_{1}, \ldots, v_{n}$. We denote the extra vertex adjacent to the cycle by $v_{0}$. Since $W_{3}$ is just the complete graph $K_{4}$, it has optimal span $3 k$. Next we consider $W_{4}$.

Proposition 5.I. For real $k \geq 0$, we have

$$
\lambda\left(W_{4} ; k, 1\right)= \begin{cases}k+1 & \text { if } 0 \leq k \leq \frac{1}{3} \\ 4 k & \text { if } \frac{1}{3} \leq k \leq 1 \\ 2 k+2 & \text { if } k \geq 1\end{cases}
$$

Proof. The upper bound is attained by the following labelings $f$, in which we give the label of the central vertex, $f\left(v_{0}\right)$, first followed after a semicolon by the labels going around the $n$-cycle:

$$
\begin{cases}(2 k ; 0, k, 1, k+1) & \text { if } \quad 0 \leq k \leq \frac{1}{3} \\ (2 k ; 0, k, 3 k, 4 k) & \text { if } \quad \frac{1}{3} \leq k \leq 1 \\ (0 ; k, 2 k+1, k+1,2 k+2) & \text { if } \quad k \geq 1\end{cases}
$$

We must verify this is also a lower bound for all $k$. For $0 \leq k \leq \frac{1}{3}$, we have $\lambda\left(W_{4} ; k, 1\right) \geq \lambda\left(C_{4} ; k, 1\right)=k+1$.

For $\frac{1}{3} \leq k \leq 1$, any two labels must differ by at least $k$, so that the span of an $L(k, 1)$-labeling is at least $4 k$. It remains to treat large $k$.

Claim 5.2. For $k \geq 1, \lambda\left(W_{4} ; k, 1\right) \geq 2 k+2$.

Proof. Let $f$ be an optimal labeling as in the $D$-Set Theorem. Any three vertices in $W_{4}$ induce either a path $P_{3}$, which has span $k+1$, or a cycle $C_{3}$, which has span $2 k \geq k+1$. So at most two vertices have labels in the interval $[0, k+1)$, and 
at most two have labels in $[k+1,2 k+2)$. Hence, some label is at least $2 k+2$, and so is the span of $f$, proving the claim and proposition.

For $n \geq 5$, we split according to whether it is odd or even.

Proposition 5.3. Let $n$ be an odd integer $\geq 5$. For real $k \geq 0$, we have

$$
\lambda\left(W_{n} ; k, 1\right)= \begin{cases}\frac{n-1}{2} & \text { if } 0 \leq k \leq \frac{1}{3} \\ 3 k+\frac{n-3}{2} & \text { if } \frac{1}{3} \leq k \leq \frac{1}{2} \\ n k & \text { if } \frac{1}{2} \leq k \leq 1 \\ k+n-1 & \text { if } 1 \leq k \leq \frac{n-1}{2} \\ 3 k & \text { if } k \geq \frac{n-1}{2} .\end{cases}
$$

Proof. The upper bound is attained by these labelings $f$, in which $f\left(v_{0}\right)$ is listed first:

For $0 \leq k \leq \frac{1}{3}$,

$$
\left(2 k ; 0, k, 1, k+1,2, k+2,3, k+3, \ldots, \frac{n-3}{2}, k+\frac{n-3}{2}, \frac{n-1}{2}\right) ;
$$

for $\frac{1}{3} \leq k \leq \frac{1}{2}$

$$
\left(2 k ; 0, k, 3 k, 4 k, 3 k+1,4 k+1,3 k+2,4 k+2, \ldots, 4 k+\frac{n-5}{2}, 3 k+\frac{n-3}{2}\right) ;
$$

for $\frac{1}{2} \leq k \leq 1$,

$$
(0 ; k, 2 k, 3 k, \ldots, n k)
$$

for $1 \leq k \leq \frac{n-1}{2}$

$\left(0 ; k, k+\frac{n+1}{2}, k+1, k+\frac{n+3}{2}, k+2, k+\frac{n+5}{2}, \ldots, k+\frac{n-3}{2}, k+n-1, k+\frac{n-1}{2}\right) ;$

for $k \geq \frac{n-1}{2}$,

$\left(0 ; k, 2 k+1, k+1,2 k+2, k+2,2 k+3, \ldots, k+\frac{n-5}{2}, 2 k+\frac{n-3}{2}, k+\frac{n-3}{2}, 3 k, 2 k\right)$.

We need to verify that these values are lower bounds. Let $f$ be an optimal labeling as in the $D$-Set Theorem. We begin with small $k$.

Claim 5.4. Let $n \geq 5$ be odd. For $0 \leq k \leq \frac{1}{3}, \lambda\left(W_{n} ; k, 1\right) \geq \frac{n-1}{2}$. 
Proof. Of the $n$ vertices on the outer cycle, no three can be in the same interval $[i, i+1)$, since some two of any three vertices on the cycle are at distance 2 in $W_{n}$. Thus, some vertex on the cycle has label outside $\left[0, \frac{n-1}{2}\right)$, so the span of $f$ is at least $\frac{n-1}{2}$, proving the Claim.

Claim 5.5. Let $n \geq 5$ be odd. For $\frac{1}{3} \leq k \leq \frac{1}{2}, \lambda\left(W_{n} ; k, 1\right) \geq 3 k+\frac{n-3}{2}$.

Proof. Using the distance conditions, all vertices with labels in the interval $[0,1)$ are mutually adjacent. So there are at most three of them, and, if there are three, one of them must be the center, $v_{0}$ (and $k<1 / 2$ ). The same is true for all of the intervals $I_{i}:=[i, i+1), 0 \leq i \leq \frac{n-3}{2}$. This means we can have at most $n$ vertices with labels in $\left[0, \frac{n-1}{2}\right)$, so that some vertex $w$ has label at least $\frac{n-1}{2}$. If there are two such vertices, the greatest label is at least $k+\frac{n-1}{2} \geq 3 k+\frac{n-3}{2}$, the desired bound. If there is only one such vertex, we must look more closely: Some interval $\left[j, j+1\right.$ ) must contain labels for three vertices (one of which is $v_{0}$ ), while the others have just two each. The greatest label in $[j, j+1)$ is at least $2 k+j$. The two labels in $[j+1, j+2)$ are then at least $k$ greater, $3 k+j$. The two labels in $[j+2, j+3)$ are at least $3 k+j+1$, because each represents a vertex in the $n$-cycle that is distance 2 from one or both vertices with labels in $[j+1, j+2)$. Repeating this idea, we eventually find that the label $f(w) \geq 3 k+\frac{n-3}{2}$, proving the Claim.

For $\frac{1}{2} \leq k \leq 1$, the lower bound is easy: Since any two vertices have labels at least $k$ apart (as $k \leq 1$ ), and there are $n+1$ vertices, the optimal span is at least $n k$.

For $1 \leq k \leq \frac{n-1}{2}$, any pair of labels in some optimal labeling $f$ (as in the $D$-Set Theorem) differ by at least $\min \{k, 1\}=1$. Suppose the labels are $0=$ $x_{0}<x_{1}<x_{2} \cdots<x_{n}$. Since $v_{0}$ is adjacent to every other vertex, there exists $i$ such that $x_{i+1}-x_{i} \geq k$, hence the span $x_{n} \geq k+n-1$, as claimed.

Finally, we consider large $k$.

Claim 5.6. Let $n \geq 5$ be odd. For $k \geq \frac{n-1}{2}, \lambda\left(W_{n} ; k, 1\right) \geq 3 k$.

Proof. Since $W_{n}$ has diameter 2 , no two adjacent vertices have labels in $[0, k)$. That is, the vertices with labels in $[0, k)$ form an independent set, and the same is true for the intervals $[k, 2 k)$ and $[2 k, 3 k)$. Since $W_{n}$ has chromatic number 4 , some vertex must have a larger label, which is at least $3 k$, so $\lambda\left(W_{n} ; k, 1\right) \geq 3 k$. This proves the claim and proposition.

Having completed the proof for odd $n$, it remains to treat even $n \geq 5$. 
Proposition 5.7. Let $n$ be an even integer $\geq 5$. For real $k \geq 0$, we have

$$
\lambda\left(W_{n} ; k, 1\right)= \begin{cases}k+\frac{n}{2}-1 & \text { if } \quad 0 \leq k \leq \frac{1}{3} \\ 4 k+\frac{n}{2}-2 & \text { if } \frac{1}{3} \leq k \leq \frac{1}{2} \\ n k & \text { if } \frac{1}{2} \leq k \leq 1 \\ k+n-1 & \text { if } 1 \leq k \leq \frac{n}{2}-1 \\ 2 k+\frac{n}{2} & \text { if } \quad k \geq \frac{n}{2}-1 .\end{cases}
$$

Proof. The upper bound is attained by these labelings $f$, in which $f\left(v_{0}\right)$ is listed first:

For $0 \leq k \leq \frac{1}{3}$,

$$
\left(2 k ; 0, k, 1, k+1,2, k+2,3, k+3, \ldots, \frac{n}{2}-1, k+\frac{n}{2}-1\right)
$$

for $\frac{1}{3} \leq k \leq \frac{1}{2}$

$$
\left(2 k ; 0, k, 3 k, 4 k, 3 k+1,4 k+1,3 k+2,4 k+2, \ldots, 3 k+\frac{n}{2}-2,4 k+\frac{n}{2}-2\right) ;
$$

for $\frac{1}{2} \leq k \leq 1$,

$$
(0 ; k, 2 k, 3 k, \ldots, n k)
$$

for $1 \leq k \leq \frac{n-1}{2}$

$$
\left(0 ; k, k+\frac{n}{2}, k+1, k+\frac{n}{2}+1, k+2, k+\frac{n}{2}+2, \ldots, k+\frac{n}{2}-1, k+n-1\right) ;
$$

for $k \geq \frac{n-1}{2}$,

$$
\left(0 ; k, 2 k+1, k+1,2 k+2, k+2,2 k+3, \ldots, k+\frac{n}{2}-1,2 k+\frac{n}{2}\right) .
$$

We now prove that the formulas above are lower bounds. As usual, assume $f$ is an optimal labeling as in the $D$-Set Theorem. Begin with small $k$.

Claim 5.8. Let $n \geq 5$ be even. For $0 \leq k \leq \frac{1}{3}, \lambda\left(W_{n} ; k, 1\right) \geq k+\frac{n}{2}-1$.

Proof. Because $W_{n}$ has diameter 2 , at most one vertex $v_{i}$ has label in $[0, k)$. If $i$ is even, then all of the vertices in the independent set $I:=\left\{v_{1}, v_{3}, v_{5}, \ldots, v_{n-1}\right\}$ have labels at least $k$. If $i$ is odd, instead use $I:=\left\{v_{2}, v_{4}, v_{6}\right.$, $\left.\ldots, v_{n}\right\}$. Any two vertices in $I$ are at distance 2 , so their labels differ by at least 1 . Thus, the labels for $I$ have span at least $|I|-1$, and the span of $f$ is at least $k+|I|-1=k+\frac{n}{2}-1$, proving the claim. 
Claim 5.9. Let $n \geq 5$ be even. For $\frac{1}{3} \leq k \leq \frac{1}{2}$, we have $\lambda\left(W_{n} ; k, 1\right) \geq 4 k+\frac{n}{2}-2$.

Proof. By the upper bound, $f$ has span at most $\frac{n}{2}$ (note that $k \leq \frac{1}{2}$ ). Similar to the proof of Claim 3 above, we see that each interval $I_{i}:=[i, i+1), 0 \leq i \leq \frac{n-2}{2}$, contains labels for a set of two mutually adjacent vertices, except that for one value $j, I_{j}$ contains labels for three vertices, one of which is $v_{0}$. The greatest of the three labels in $I_{j}$ is at least $2 k+j$.

If $j=\frac{n-2}{2}$, then we have a label that is at least $4 k+\frac{n}{2}-2$, the bound we seek.

On the other hand, suppose $j<\frac{n-2}{2}$. Then the two labels in $I_{j+1}$ are at least $k$ greater, so they are at least $3 k+j$. We find successively (similar to the proof of Claim 3) that the two labels in $I_{j+2}$ are at least $3 k+j+1$, and so on, and at least $3 k+\frac{n-2}{2}-1$ in $I_{\frac{n}{2}-2}$. The greater of the two labels in the last interval is then at least $4 k+\frac{n}{2}-2$, the desired bound, proving the claim.

For $\frac{1}{2} \leq k \leq \frac{n}{2}-1$, the lower bounds follow by the same arguments as for Proposition 5.3. It remains to treat large $k$.

Claim 5.10. Let $n \geq 5$ be even. For $k \geq \frac{n}{2}-1$, we have $\lambda\left(W_{n} ; k, 1\right) \geq 2 k+\frac{n}{2}$.

Proof. The labels used by $f$ are distinct and separated by at least 1 , except that $f\left(v_{0}\right)$ is separated by at least $k$ from the others. If $f\left(v_{0}\right)$ is neither the least nor the greatest label, then the span of $f$ must be at least $2 k+n-2 \geq 2 k+\frac{n}{2}$, which is the desired bound.

Hence, suppose $f\left(v_{0}\right)$ is an extreme value, say it is 0 . (If instead it is the greatest value, the span of $f$, just take the complementary labeling.) If $f$ has more than $\frac{n}{2}$ labels that are at least $2 k$, then the greatest of them must be at least $2 k+\frac{n}{2}$, which is the bound we want.

Else, there are at most $\frac{n}{2}$ labels that are at least $2 k$. Then we have at least $\frac{n}{2}$ labels in $[k, 2 k)$. Indeed, we have exactly this many labels in $[k, 2 k)$ due to the fact that the corresponding vertices must be independent in $W_{n}$. Hence, we have exactly $\frac{n}{2}$ labels that are at least $2 k$. The greatest label in $[k, 2 k)$ must be at least $k+\frac{n}{2}-1$, while its two neighbors on the cycle in $W_{n}$ must both have labels that are at least $2 k+\frac{n}{2}-1$; the greater one is at least $2 k+\frac{n}{2}$. Hence the span of $f$ is at least this value. This proves the claim and proposition.

This completes the proof of Theorem 2.3.

Acknowledgements. The authors wish to thank Julie Emery and Katherine Heller, who, as undergraduates, carefully worked through the proofs for paths and cycles during summer 2003. This was part of a summer research project under the direction of the 
authors. Research for this paper was supported in part by NSF grants DMS-0072187 and DMS-0302307 and by the Industrial Mathematics Institute at USC. The principal results here were also described in the second author's dissertation [Jin 05].

\section{References}

[Anand et al. 03] S. Anand, A. Sridharan, and K. N. Sivarajan. "Performance Analysis of Channelized Cellular Systems with Dynamic Channel Allocation." IEEE Transactions on Vehicular Technology 52:4 (2003), 847-859.

[Bertossi et al. 03] A. A. Bertossi, C. M. Pinotti, and Richard B. Tan. "Channel Assignment with Separation for Inference Avoidance in Wireless Networks." IEEE Trans. Parallel Distrib. Systems 14:3 (2003), 222-235.

[Calamoneri 06] T. Calamoneri. "The $L(h, k)$-Labelling Problem: An Annotated Bibliography." Online survey updated and posted at http://www.dsi.uniroma1.it/ $\sim$ calamo/PDF-FILES/survey.pdf, 2006.

[Georges and Mauro 95] J. P. Georges and D. W. Mauro. "Generalized Vertex Labelings with a Condition at Distance Two." Congress. Numer. 109 (1995), 141-159.

[Griggs 00] J. R. Griggs. "Author/Judge's Commentary: The Outstanding Channel Assignment Papers." UMAP J. 21 (2000), 379-386.

[Griggs and Jin 05] J. R. Griggs and X. T. Jin. "Optimal Channel Assignments for Lattices with Conditions at Distance Two." In Proceedings of the 19th IEEE International Parallel and Distributed Processing Symposium, Workshop 12, Volume 13, 8 pp. Los Alamitos, CA: IEEE Computer Society, 2005.

[Griggs and Jin 06] J. R. Griggs and X. T. Jin. "Real Number Graph Labellings with Distance Conditions." SIAM J. Discrete Math. 20 (2006), 302-327.

[Griggs and Jin 07] J. R. Griggs and X. T. Jin. "Recent Progress in Mathematics and Engineering on Optimal Graph Labellings with Distance Conditions." J. Combin. Optimiz. 14 (2007), 249-257.

[Griggs and Jin 08] J. R. Griggs and X. T. Jin. "Real Number Channel Assignments for Lattices." SIAM J. on Discrete Math. 22:3 (2008), 996-1021.

[Griggs and Král', to appear] J. R. Griggs and D. Král'. "Graph Labellings with Variable Weights, A Survey." Discrete Appl. Math., to appear.

[Griggs and Yeh 92] J. R. Griggs and R. K. Yeh. "Labelling Graphs with a Condition at Distance 2." SIAM J. Disc. Math. 5 (1992), 586-595.

[Hale 80] W. K. Hale. "Frequency Assignment: Theory and Applications." Proc. IEEE 68 (1980), 1497-1514.

[Jin 05] X. T. Jin. "Real Number Graph Labeling with Distance Conditions.' Ph.D. Dissertation, University of South Carolina, August, 2005.

[Král' 06] D. Král'. "The Channel Assignment Problem with Variable Weights. SIAM J. Discrete Math. 20 (2006), 690-704. 
[Roberts 03] F. S. Roberts. "Working Group Agenda, DIMACS/DIMATIA/Renyi Working Group on Graph Colorings and Their Generalizations." Available online at http://dimacs.rutgers.edu/Workshops/GraphColor/main.html, 2003.

[Sarkar and Sivarajan 02] S. Sarkar and K. N. Sivarajan. "Channel Assignment Algorithms Satisfying Cochannel and Adjacent Channel Reuse." IEEE Transactions on Vehicular Technology 51:5 (2002), 954-967.

Jerrold R. Griggs, Department of Mathematics, University of South Carolina, Columbia, SC 29208 (griggs@math.sc.edu)

Xiaohua Teresa Jin, Department of Electrical Engineering, University of South Carolina,

Columbia, SC 29208 (xhxh77@hotmail.com)

Received May 4, 2006; accepted in revised form October 5, 2007. 